Check for updates

Cite this: RSC Adv., 2018, 8, 34047

\title{
Effects of proton conduction on dielectric properties of peptides $\dagger$
}

\author{
Taehoon Sung, ${ }^{\text {ab }}$ Seok Daniel Namgung, ${ }^{\text {ab }}$ Jaehun Lee, (D) ${ }^{c}$ Ik Rang Choe, ${ }^{c}$ \\ Ki Tae Nam (D) *c and Jang-Yeon Kwon (iD *ab
}

Peptides have been overlooked for their use in the field of electronics, even though they are one of the most commonly found bio-induced materials, and are not only easy to mass-produce but also exhibit a high dielectric constant. Additionally, unlike proteins, which are gaining considerable interest with materials researchers, peptides are much simpler, rendering their original characteristics easier to maintain without significant alteration of their structure. On the other hand, proteins tend to deform due to their susceptibility to environmental changes. Combining such superb dielectric properties with their relatively stable nature, peptides could be utilized as a component of electronic devices ranging from basic capacitors to more complex thin-film transistors. In this paper, a peptide chain (YYACAYY) composed of tyrosine, alanine, and cysteine was extensively studied using an impedance analyzer to determine its innate charge movement mechanism in order to extend our understanding of the electric properties of peptides. The movement of mobile protons inside the peptide insulator was found to be the source of the high relative permittivity of the peptide insulator, and the dielectric constant of the peptide insulator was found to be over 17 in humid conditions. By widening the understanding of the dielectric properties of the peptide insulator, it is expected that the peptide can be further utilized as an insulator in various electronic devices.

Received 19th July 2018

Accepted 26th September 2018

DOI: $10.1039 / c 8 r a 06121 a$

rsc.li/rsc-advances level, and mostly focused on revealing the underlying proton conduction mechanism of the molecules. ${ }^{7}$ Additionally, recent research on the bulk protonic conduction found in most biomolecules demonstrates that such bio-molecules exhibit proton conductivity, ${ }^{6,8-11}$ although little effort has been made in utilizing the proton conductivity in actual electronic devices.

Therefore, this paper focuses on studying the dielectric property of the suggested peptide molecules with respect to their proton conductivity, and on the applicability of the peptide as a dielectric layer that can be utilized in many different types of electronic devices.

The peptide chain (YYACAYY) used in this research is composed of tyrosine (Y), alanine (A), and cysteine (C), as shown in Fig. 1. This specific peptide sequence contains functional groups, including $-\mathrm{OH},-\mathrm{COOH},-\mathrm{NH}_{2}$, and $-\mathrm{SH}$, which are known to be hydrophilic and to exhibit good ionic<smiles>CC(NC(=O)C(Cc1ccc(O)cc1)NC(=O)C(N)Cc1ccc(O)cc1)C(=O)NC(CS)C(=O)NC(C)C(=O)NC(Cc1ccc(O)cc1)C(=O)NC(Cc1ccc(O)cc1)C(=O)O</smiles>

Fig. 1 Chemical Structure of the YYACAYY peptide.
${ }^{a}$ School of Integrated Technology, Yonsei University, Incheon 21983, Republic of Korea ${ }^{5}$ Yonsei Institute of Convergence Technology, Yonsei University, Incheon 21983, Republic of Korea.E-mail: jangyeon@yonsei.ac.kr

'Department of Materials Science and Engineering, Seoul National University, Seoul 08826, Republic of Korea. E-mail: nkitae@snu.ac.kr

$\dagger$ Electronic supplementary information (ESI) available. See DOI: $10.1039 / \mathrm{c} 8 \mathrm{ra06121a}$ 
conductivity. ${ }^{\mathbf{1 2 , 1 3}}$ There are few amino acids that take critical role in proton conduction in biological systems. Especially, amino acids with phenol groups, for example tyrosine, are known to be an important constituents that construct proton-coupled electron transfer observable in ribonucleotide reductase ${ }^{\mathbf{1 4}}$ and photosystem $\mathrm{II}^{15}$ in association with inorganic clusters. In addition, tyrosine is a precursor of eumelanins, ${ }^{\mathbf{1 6}}$ which exhibit humidity-dependent high proton conductivity. Such protonic conductivity is due to the absorption of water molecules introduced to the peptide film from the ambient atmosphere. ${ }^{5,10,17}$ Furthermore, the absorbed water molecules constitute a hydrogen bond network, where protons may easily hop from one place to another as described by the Grotthuss mechanism, ${ }^{11,18,19}$ and the proton conductivity present inside the peptide may enhance the dielectric properties of the peptide, since protons, which cannot pass through metal electrodes, may form an electrical double layer (EDL) at the interface, therefore increasing the relative permittivity of the material. ${ }^{20}$

Additionally, unlike proteins that are susceptible to environmental changes due to their complex bonding and structure, the peptides remain fairly stable during conventional devicefabrication processes, which often induce heat and relatively strong organic solvents. Furthermore, short-chain peptides are relatively small and stable molecules that are easier to synthesize with well-known processes. ${ }^{21}$ Also, the specific peptide sequence used in this research can be formed into a large-area film and its resistivity can easily be tuned..$^{22,23}$ Such flexibility of the peptide sequence is expected to be helpful in future research, as the mechanical and electrical properties of the peptide can be altered in researcher's favor.

To ease the fabrication process and to prove that this peptide insulator can be integrated into conventional electronic devices, conventional thin-film fabrication methods such as spincoating, sputtering, and evaporating techniques were utilized in the fabrication of the test samples. The 3-D schematics and microscopic images of the fabricated devices are shown in Fig. 2. Fig. 2b shows the 3-D schematics of the metal-insulatormetal (MIM) samples that are used in the impedance and breakdown analyses, while Fig. 2c shows the schematics of the Thin Film Transistor (TFT) samples that are fabricated with an In-Ga-Zn-O (IGZO) active layer and a peptide insulator. From the optical microscopic image in Fig. $2 \mathrm{~d}$ and the side-view using Transmission Electron Microscopy (TEM) in Fig. 2e, successful fabrication of the TFT samples was confirmed.

Using the fabricated MIM and TFT samples, the peptides were analyzed using various electronic analysis techniques involving an impedance analyzer and a semiconductor analyzer. From the analysis, the relative permittivity of the peptide insulator was found to be over 20 in humid conditions. In addition, the origin of the high relative permittivity and internal movement of the protons inside the peptide insulator could be examined, and the relationship between the protonic movement and the relative permittivity could be found.

Based on the understanding from studying the property of the peptide insulator, it could also be integrated into a functioning oxide TFT to illustrate the applicability of the peptide insulator. The resulting peptide-integrated TFT showed superior characteristics in terms of on-current and on-off ratio due to large dielectric constant of the peptide insulator. Combined with the understanding of the protonic movement reported in this paper, it is believed that the electrical property of the peptide insulator can be further enhanced to provide bioinduced high-k insulator that can be employed in many electronic applications.

\section{Experimental}

\subsection{Sample preparation}

2.1.1. Deposition of gate dielectrics. The YYACAYY peptide was prepared and dissolved into Trifluoroacetic Acid (TFA), with a

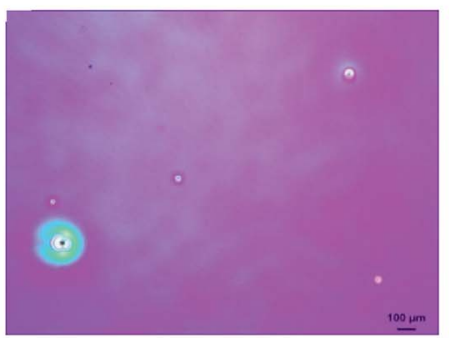

c

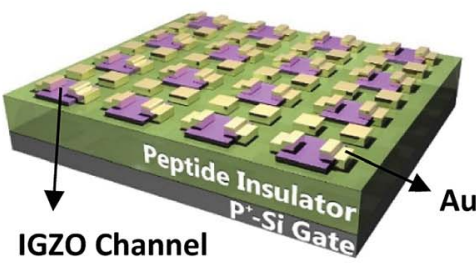

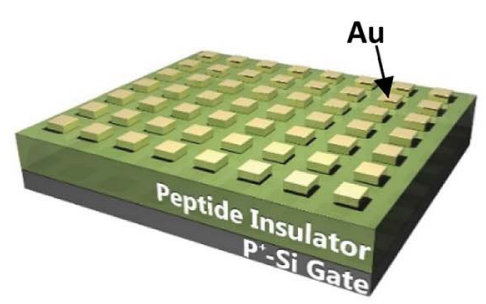

d

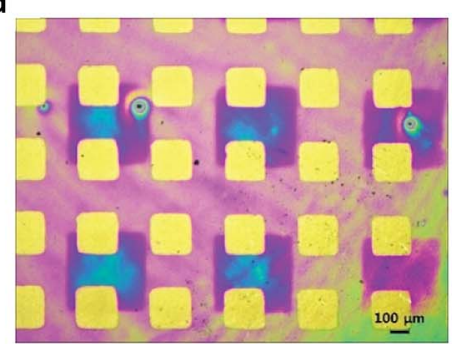

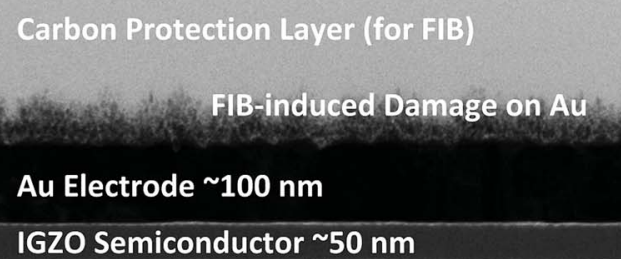

Peptide Insulator $\sim \mathbf{4 0 0 ~} \mathrm{nm}$
$200 \mathrm{~nm}$

$\mathbf{P}^{++}$-Si Substrate

Fig. 2 (a) Optical microscopic image of the RH-controlled peptide film. 3-D structure of (b) MIM sample and (c) TFT sample. (d) Top-view optical microscopic image and (e) side-view TEM image of IGZO TFTs with the peptide insulator. 
the concentration of $4 \mathrm{wt} \%$. The peptide solution was then spincoated at the rotating speed of 4000 RPM for 60 seconds above a gate electrode, which is a $\mathrm{p}^{++}$-Si substrate, while controlling the relative humidity at the time of the coating. Spin-coating was chosen to easily control the thickness of the peptide by changing the concentration of the peptide solution. The relationship between the thickness of the peptide and the concentration of the peptide solution was linearly proportional and can be found in Fig. S1a in ESI. $\dagger$ The thickness was optimized at $400 \mathrm{~nm}$ to gain dielectric strength and to avoid possible thickness variation due to the initial thickness being too thin. The spin-coated peptide is amorphous as the TEM image in Fig. 2e and SAED pattern in Fig. S3 in ESI $\dagger$ show. Optical striation was found in the samples that were spin-coated at low Relative Humidity (RH) conditions (approximately 45\%), while no striation was found in the samples that were spin-coated at high $\mathrm{RH}$ conditions (approximately 80\%). Regardless of the $\mathrm{RH}$ condition at the time of the spin-coating, the peptide layer showed excellent surface morphology when measured with atomic force microscopy (AFM) with the root mean square values of $0.189 \mathrm{~nm}$ and $0.193 \mathrm{~nm}$ for the $\mathrm{RH}$-controlled and noncontrolled samples, respectively.

In the case of the $\mathrm{SiO}_{2}, 400 \mathrm{~nm}$-thick Plasma-Enhanced Chemical Vapor Deposition (PE-CVD) $\mathrm{SiO}_{2}$ and $100 \mathrm{~nm}$-thick thermally grown $\mathrm{SiO}_{2}$ were used for the MIM and TFT samples, respectively. Since the capacitance is greatly influenced by the thickness of the insulator, $\mathrm{SiO}_{2}$ with the same thickness as the peptide insulator was utilized during impedance analysis. However, in order to discount the effect of annealing process on the TFT samples, neither the peptide TFT nor the reference $\mathrm{SiO}_{2}$ TFT were annealed. Therefore, thermally grown $\mathrm{SiO}_{2}$ with better characteristics was used instead of the same-thickness PE-CVD $\mathrm{SiO}_{2}$ to optimize the performance of the reference $\mathrm{SiO}_{2}$ TFT.

For the $400 \mathrm{~nm}$-thick PE-CVD $\mathrm{SiO}_{2}$, the Radio Frequency (RF) power was set as $100 \mathrm{~W}$ at $300{ }^{\circ} \mathrm{C}$ under 950 mTorr, with gas flow rates of $30 \mathrm{sccm}, 100 \mathrm{sccm}$, and $100 \mathrm{sccm}$ for $\mathrm{SiH}_{4}, \mathrm{~N}_{2} \mathrm{O}$, and $\mathrm{Ar}$, respectively. The refractive index was measured from an ellipsometer as 1.451 .

For the $100 \mathrm{~nm}$-thick thermally grown $\mathrm{SiO}_{2}$, pre-fabricated $100 \mathrm{~nm}$-thick thermally grown oxides on a $\mathrm{P}^{++}$-Si substrate were used, which were produced from Dasom RMS in South Korea.

2.1.2. Deposition of channel layer IGZO channel. An IGZO channel with a thickness of $50 \mathrm{~nm}$ was deposited above the gate dielectric layer with an RF sputter. In order to avoid any chemical damage that might be introduced during conventional photolithography where numerous chemicals are used, a shadow mask with $400 \mu \mathrm{m} \times 400 \mu \mathrm{m}$ square holes was used to pattern the IGZO channel. The RF power was set to $100 \mathrm{~W}$ with gas flow rates of $30 \mathrm{sccm}$ and $0.5 \mathrm{sccm}$ for $\mathrm{Ar}$ and $\mathrm{O}_{2}$, respectively. The pressure was set at 5 mTorr.

2.1.3. Deposition of electrodes. After the deposition of the channel layer, $200 \mathrm{~nm}$-thick Au was thermally evaporated onto the channel layer using a shadow mask with $200 \mu \mathrm{m} \times 200 \mu \mathrm{m}$ square holes, which were manually aligned with the channels using an optical microscope.

\subsection{Characterization}

2.2.1. Impedance analysis. Impedance of the peptide and the reference $\mathrm{SiO}_{2}$ insulators was studied in the frequency range of $20 \mathrm{~Hz}$ to $1 \mathrm{MHz}$ using an impedance analyzer (Keysight Technologies, E4990A, USA), and the related parameters, such as capacitance, phase angle difference, and real and imaginary impedance were extracted simultaneously. For every frequency, an AC perturbation voltage of $500 \mathrm{mV}$ and a DC bias voltage of $-1 \mathrm{~V}$ were used. Measurement was performed in a chamber at room temperature, where vacuum was applied for the $\mathrm{RH} 0 \%$ condition. For the RH 50\% and $80 \%$ conditions, a humidifier was used to add the desired amount of moisture to the chamber, which was under atmospheric pressure at room temperature. The chamber was then sealed to contain the moisture inside. Additionally, for each humidity condition, measurement was delayed for 1 hour to allow moisture to diffuse throughout the chamber.

2.2.2. Equivalent circuit fitting. Experimental data from the impedance analysis were analyzed using an analysis tool (Scribner, ZView, USA). The calculated chi-square values for $0 \%$ $\mathrm{RH}, 50 \% \mathrm{RH}$, and $80 \% \mathrm{RH}$ were $0.011,0.016$, and 0.006 , respectively.

2.2.3. Resistivity and breakdown measurement. Similar to the impedance analysis, the chamber was used to maintain the desired humidity condition. A semiconductor analyzer (Keithley Instruments, 4200-SCS, USA) was used to measure the current according to the voltage applied. During the measurement of the breakdown voltage and resistivity, the compliance current was set to $0.01 \mathrm{~A}$.

2.2.4. TFT characteristics measurement. The chamber was also used to maintain the desired humidity condition. A semiconductor analyzer (Keithley Instruments, 4200-SCS, USA) was used to apply a fixed drain voltage of $10 \mathrm{~V}$ and to sweep the gate voltage from $-10 \mathrm{~V}$ to $40 \mathrm{~V}$ while simultaneously measuring the drain current.

\section{Results \& discussion}

\subsection{Impedance analysis}

As the first part of the experiment, the impedance analyzer was utilized to measure the impedance in terms of the capacitance and phase angle, as well as the real and imaginary impedance of the peptide insulator under various alternating current (AC) perturbation frequency and $\mathrm{RH}$ conditions to understand the conduction mechanism of the peptides. ${ }^{24}$ From the results of impedance analysis, the true nature of the mobile charges that reside inside the peptide insulator were expected to be discovered, ${ }^{25}$ and based on the understanding on the movement of mobile charges, the source of the high relative permittivity of the peptides in relation to the protonic behavior can be studied. ${ }^{20}$

This section analyzes the Metal-Insulator-Metal (MIM) samples, which are composed of an insulator layer sandwiched between the top and the bottom electrodes. Since the MIM samples are basically parallel-plate capacitors, the capacitance of the peptide insulator was measured first by changing both 
the $\mathrm{RH}$ conditions and the frequency of AC perturbation. The $\mathrm{AC}$ perturbation is an AC signal superimposed on the direct current (DC) signal and is usually in the range of less than $1 \mathrm{~V}$. By altering the relative electric potential of the top and bottom electrodes, the AC perturbation causes mobile charges to move in and out of the capacitor. Also, by measuring the number of charges that respond, the capacitance can be measured.

3.1.1. Capacitance and phase angle. This section shows that the peptide insulator exhibits high relative permittivity at low frequency range. Furthermore, the results from the phase angle analysis indicate the presence of mobile charges inside the peptide insulator.

Fig. 3a shows the relationship between the capacitance of the peptide insulator and the frequency of AC perturbation. The level of AC perturbation was $500 \mathrm{mV}$, and the DC bias was set at -1 V. From the capacitance-frequency curves of the peptide insulator measured at the RH level of $50 \%$ and $80 \%$, it is found that the capacitance of the peptide insulator increased as the AC frequency decreased and as the humidity level increased. On the other hand, the capacitance of the peptide insulator measured at the $\mathrm{RH}$ level of $0 \%$ and the reference $\mathrm{SiO}_{2} \mathrm{MIM}$ sample with the dielectric thickness of $400 \mathrm{~nm}$ remain relatively constant under the entire frequency range. The corresponding dielectric constants of the peptide insulator at $20 \mathrm{~Hz}$ for $0 \% \mathrm{RH}$,
$50 \% \mathrm{RH}$ and $80 \% \mathrm{RH}$ conditions are 6.5, 7.8 and 17.2, respectively.

Also, in Fig. 3b, the graph shows the relationship between the phase angle difference and the frequency of AC perturbation. From the curves of the peptide insulator measured at 50\% $\mathrm{RH}$ and $80 \% \mathrm{RH}$, the phase angle difference between the voltage and the current of the peptide capacitor showed both capacitive and resistive behaviors, since the ideal resistor shows a phase angle difference of $0^{\circ}$ and the ideal capacitor shows a phase angle difference of $90^{\circ} .^{26}$

By combining the results shown in Fig. $3 a$ and $b$, this changing behavior can be further explained by detailed analysis on the movement of the mobile charges inside the peptide insulator.

At a high frequency range, only the electrons have sufficient mobility to react to fast-changing AC perturbation and form capacitance, while the protons barely shift their positions due to their lower mobility than that of the electrons, as shown in the diagram in Fig. 3c. This results in the capacitive behavior shown in Fig. $3 \mathrm{~b}$, since the movement of protons, or the protonic current (which shows resistive behavior) is minimized.

At a medium frequency range, the protons start responding to the AC perturbation, since the polarity of the electrical field changes to a slower speed compared to the high frequency

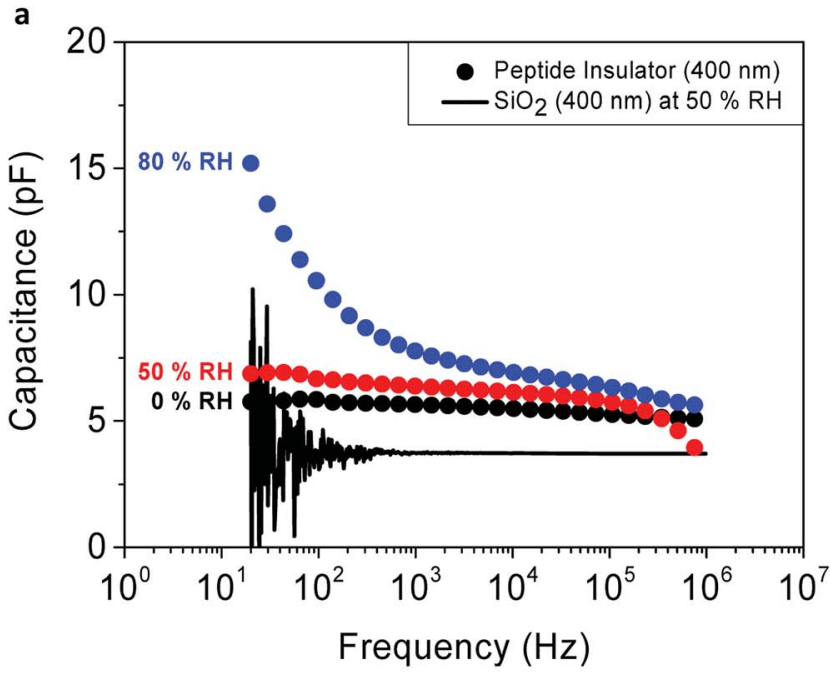

c

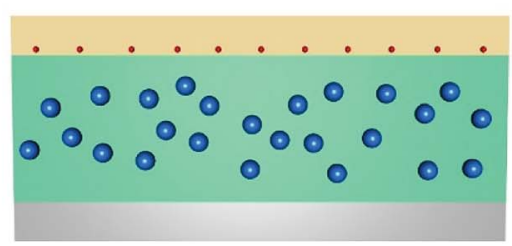

d

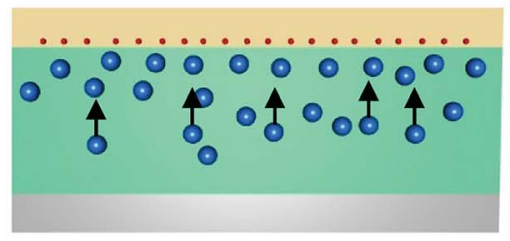

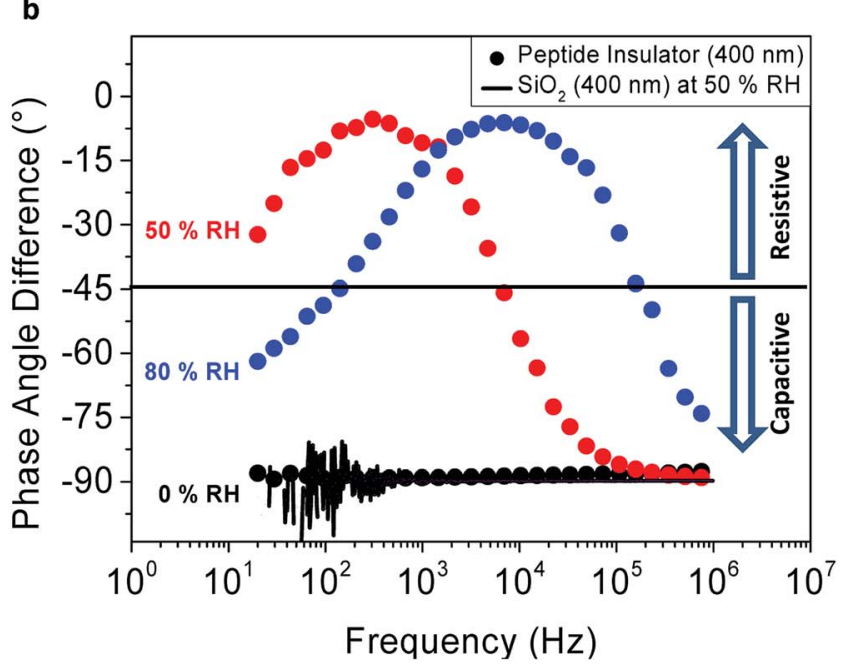

e

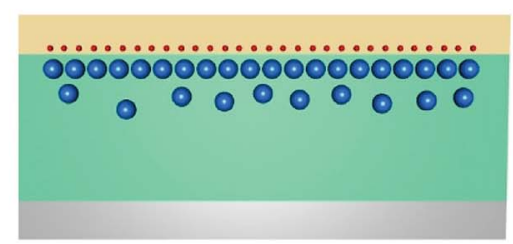

- $\quad$ : Electrons

: Protons

Fig. 3 Measurement of capacitance and phase angle of the peptide insulator. (a) Capacitance plots and (b) phase angle plots of the peptide insulator and the reference $\mathrm{SiO}_{2}$ as a function of $\mathrm{AC}$ frequency and $\mathrm{RH}$ conditions. Schematics of the behavior of protons in the peptide insulator under (c) high-frequency, (d) mid-frequency, and (e) low-frequency AC perturbation. 
range, and the protonic current flows inside the peptide insulator, as shown in Fig. 3d. However, the AC perturbation is still sufficiently fast to prevent a large portion of the protons from forming capacitance. Therefore, based on the polarity of the electrical field induced by the AC perturbation, the majority of protons continuously move vertically up and down inside the peptide insulator. Since the protonic current has dissipative characteristics, it appears as a resistive term in the phase angle difference plot in Fig. 3b.

Lastly, at low frequency range, since the protons now have a sufficient time period between the polarity shifts of the AC perturbation, the protons start contributing more to the formation of the EDL at the top or the bottom interfaces between the peptide insulator and the electrodes, by being pushed to one side of the electrodes. Their positions are thus maintained until the polarity of the AC perturbation changes and the AC perturbation exerts a sufficient opposite directional electric force to inversely push the protons to the other electrode, as shown in Fig. 3e.

In addition, the $\mathrm{RH}$ dependency of the resistive and capacitive behaviors can be observed in both Fig. 3a and b. As mentioned above, the peptide insulator may show capacitive or resistive behavior, depending on the frequency of AC perturbation and the amount of protons that react to the AC perturbation. By comparing the curves of the peptide insulator under different humidity conditions, as shown in Fig. 3b, it was found that the peptide insulator measured in a higher humidity condition exhibits more resistive behavior, implying that larger protonic current flows at the same AC frequency than the peptide insulator measured in the lower humidity condition. For example, at $10^{4} \mathrm{~Hz}$ shown in Fig. $3 \mathrm{~b}$, the peptide insulator under $80 \% \mathrm{RH}$ shows a phase angle difference of nearly $0^{\circ}$, where that of the peptide insulator under $50 \% \mathrm{RH}$ is approximately $-45^{\circ}$, and nearly $-90^{\circ}$ for the peptide insulator under $0 \% \mathrm{RH}$.

Since proton mobility is mostly affected by the fraction of absorbed water molecules that function as bridges for the protons to move around in the medium, the peptide insulator exhibits higher proton mobility at $80 \% \mathrm{RH}$, which induces the protons inside the peptide insulator to start responding to the AC perturbation, even at higher frequency, compared to the proton mobility at $50 \% \mathrm{RH}$; ultimately, very diminished protonic movements at $0 \% \mathrm{RH}$ can be inferred from the graph.

Similar to the behavior of the peptide insulator at $0 \% \mathrm{RH}$, the reference sample with the $\mathrm{SiO}_{2}$ insulator behaves only as a capacitor, even at $50 \% \mathrm{RH}$, where its phase angle difference remains at $-90^{\circ}$ for the entire frequency range. This is because the protonic conductivity of $\mathrm{SiO}_{2}$ is much less than that of the peptide insulator. This result suggests that both the electrons and the protons contribute to the formation of overall capacitance in the peptide insulator.

3.1.2. Comparison with equivalent circuit. To further identify the source of the increased capacitance of the peptide insulator, real and imaginary impedance plots of the peptide insulator were interpreted with an equivalent circuit for each $\mathrm{RH}$ condition shown in Fig. 4. The equivalent circuit is shown in
Fig. 4a and is commonly used to represent the electrolytic behavior of capacitors., ${ }^{5,27}$

In this equivalent circuit, the capacitance formed due to the electrons at each electrode was represented as the " $\mathrm{C}$ " term, and the resistance formed due to the flow of protons and electrons was represented as the " $R$ " term. The electrical double layer formed by the protons at the interface between the peptide insulator and electrodes was represented by a constant phase element (CPE) term due to its non-perfect and dissipative nature. ${ }^{20,28}$

In the experimental results represented as dots in Fig. 4a, a semicircle and low-frequency tail was observed. This is a commonly observed behavior as protons are blocked at the electrodes, ${ }^{5,12}$ since the top and the bottom electrodes of the MIM samples were fabricated using Au and highly-doped p-type Si, respectively, which are not proton-conducting materials.

From the simulated results, it was found that the phases of the CPE terms are 0.641 and 0.747 for $\mathrm{RH} 50 \%$ and $\mathrm{RH} 80 \%$ respectively. This demonstrates the dissipative property of each EDL, resulting in the slanted low-frequency tail. Also, note that the radius of each semi-circle differs with respect to the value of the resistivity. A larger resistance implies a larger radius of the semi-circle. ${ }^{8}$ Unfortunately, due to the detection limit of our equipment, a full complex plain impedance plot of the peptide

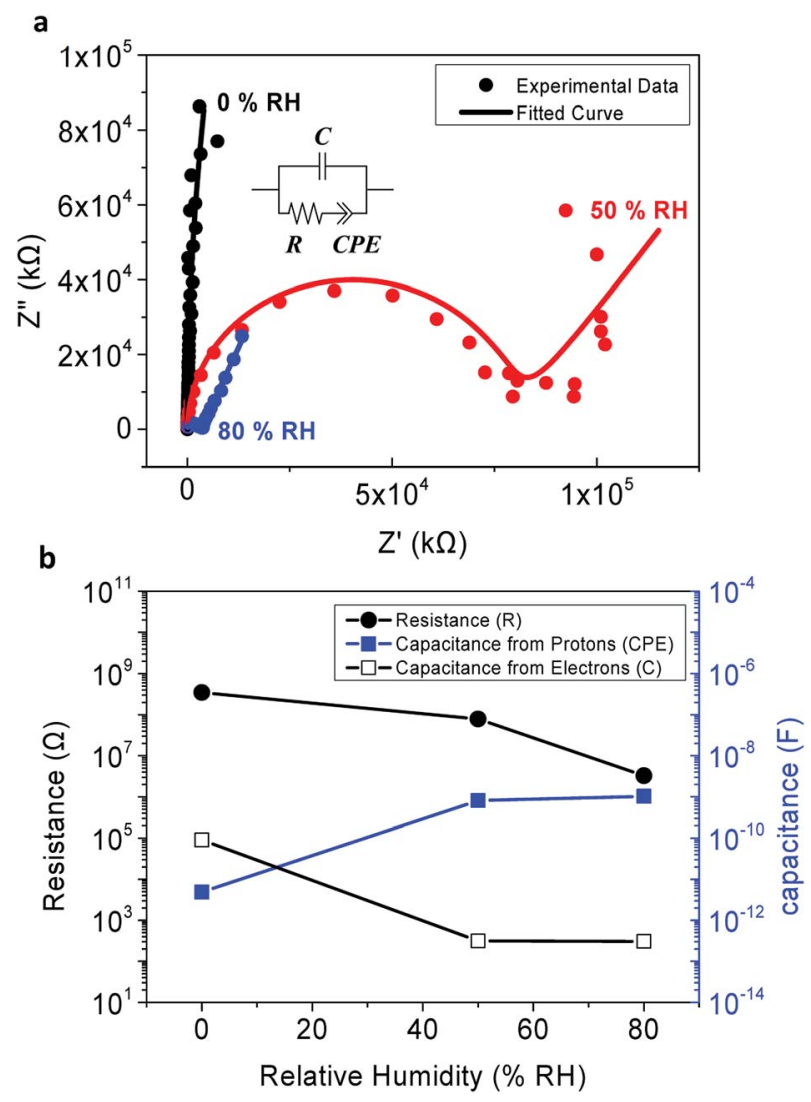

Fig. 4 Comparison of the experimental results and simulated results. (a) Complex impedance plots of the experimental results (dots), fitted curves (line), and according equivalent circuit (inset). (b) Comparison of changes in each circuit element used in the equivalent circuit as $\mathrm{RH}$ condition changed. 
insulator at $0 \% \mathrm{RH}$ was not achievable, since the peptide insulator showed very high resistance at the $\mathrm{RH}$ level of $0 \%$.

As shown in Fig. 4a, the fitted curve with the equivalent circuits agreed well with the experimental data, and such coherence implies that our equivalent circuits have accurately modeled charge movements in the peptide insulator. Therefore, it was concluded that the mobile protons, which tend to accumulate at the surface of the blocking electrode, reside inside the peptide insulator, and the protons are the main causes of the high relative permittivity of the peptide insulator.

Additionally, as shown in Fig. 4b, the tendency of increasing capacitance with respect to the increasing humidity condition, as previously observed in Fig. 3a, was again confirmed from the simulated R, C, and CPE values from the equivalent circuits. Especially, the capacitance due to electrons, which is represented by the $\mathrm{C}$ term, showed a decreasing tendency as the humidity level increased. It is believed that this is due to the increased level of leakage current which prohibits the electrons from accumulating at each side of the electrodes. Instead, the electrons further dissipate into the electrodes from the insulator or vice versa as the humidity level increases. On the other hand, the capacitance due to EDL formed by protons, which is represented as the CPE term, increased with increased level of humidity condition. As discussed earlier in the results of capacitance and phase angle measurement, this behavior can be explained by considering the increased proton conductivity at the higher humidity condition.

Since the CPE terms in the $50 \% \mathrm{RH}$ and $80 \% \mathrm{RH}$ conditions are greater than the $\mathrm{C}$ term by at least 3 orders of magnitude, it is safe to conclude that the formation of EDL by protons is the source of the high relative permittivity of the peptide insulators.

After discovering the origin of the high relative permittivity of the peptide insulator, the dielectric strength and resistivity of the peptide insulator were analyzed to determine the applicability of the peptide insulator in electronic devices.

\subsection{Dielectric strength and resistivity}

Before integrating the peptide insulator in the TFT samples, the leakage current and voltage of the induced electrical field were measured from the MIM samples to confirm that the peptide has suitable resistivity and dielectric strength to be utilized as an insulator.

In Fig. 5a, current density is shown as a function of a vertical DC electrical field induced on the peptide MIM samples. The breakdown voltage is the maximum level of electric potential difference between the top and the bottom electrodes that insulators can withstand before letting the current pass through, and is often used to represent the dielectric strength of an insulator.

The median of the electrical breakdown voltage was observed at $3.7 \mathrm{MV} \mathrm{cm}{ }^{-1}, 2.6 \mathrm{MV} \mathrm{cm}^{-1}$, and $2.15 \mathrm{MV} \mathrm{cm}^{-1}$ for $0 \% \mathrm{RH}$, $50 \% \mathrm{RH}$, and $80 \% \mathrm{RH}$, respectively. Of the ten measurements, the three curves that were closest to the median value of the electrical breakdown voltage were chosen, as shown in Fig. 5a, for each humidity level to enhance the graphical representation of the result. Such degradation of the dielectric strength in the
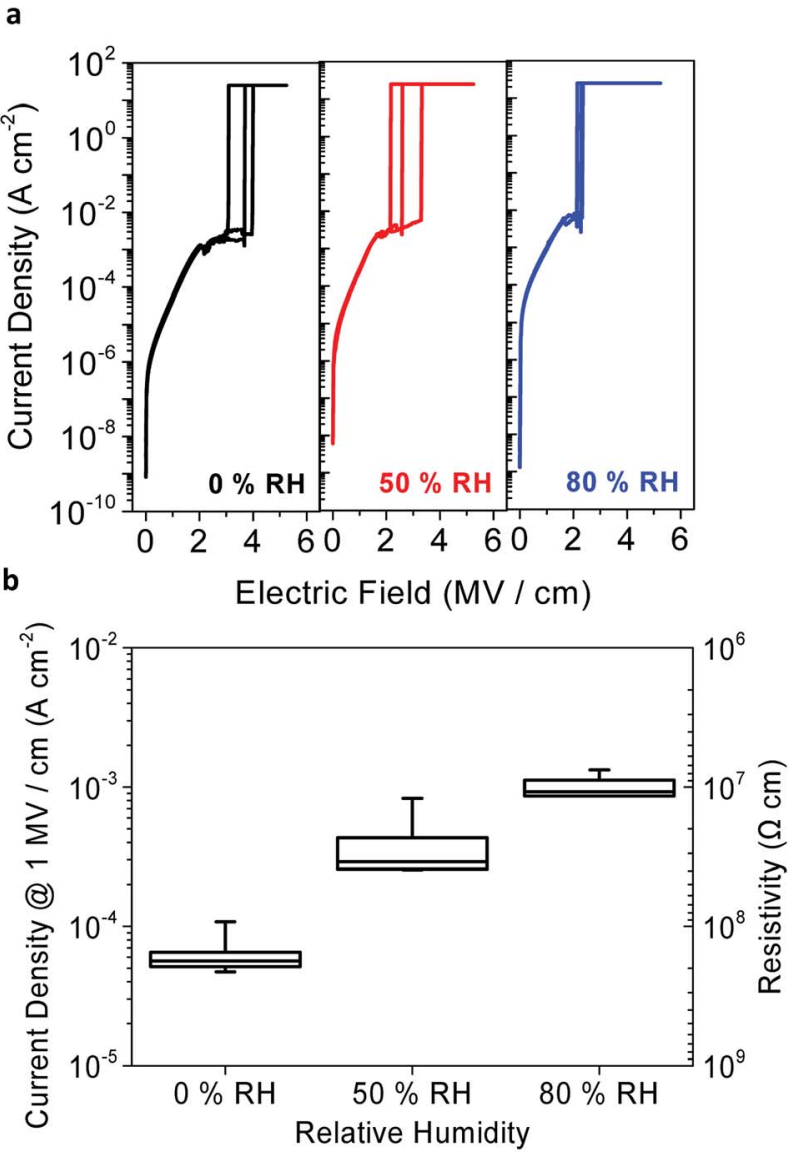

Fig. 5 Dielectric strength and resistivity of the peptide insulator. (a) Breakdown curves and (b) current density graph of the peptide insulator under various $\mathrm{RH}$ conditions.

peptide insulator at higher humidity levels is typical, and has been well explained in many other studies. ${ }^{29-31}$

Fig. 5b shows the current density and resistivity of the ten measurements for each humidity level. The current density can be converted to resistivity, and the subsequent resistivity is shown on the right axis in Fig. 5b. In terms of the leakage current, the peptide insulator exhibited relatively higher leakage current, which varied from around $10^{-4} \mathrm{~A} \mathrm{~cm}^{-2}$ to $10^{-3}$ $\mathrm{A} \mathrm{cm}^{-2}$ according to the $\mathrm{RH}$ conditions, compared to that of the reference $\mathrm{SiO}_{2}$ insulator, which was around $10^{-8} \mathrm{~A} \mathrm{~cm}^{-2}$. It is significant that the resistivity of the peptide insulator decreased at higher humidity levels, indicating that the electrical conductivity of the peptide insulator increased as the $\mathrm{RH}$ increased. Also, this result supports the simulation result shown in Fig. 4b, where the resistance represented by the $R$ term decreased with increased humidity level. This is possibly because the water molecules enhance not only the proton conduction, but also the electronic conduction. ${ }^{5}$

Since the peptide insulator with the thickness of $400 \mathrm{~nm}$ withstood at least $80 \mathrm{~V}$, which is equal to $2 \mathrm{MV} \mathrm{cm}{ }^{-1}$, and showed a resistivity larger than $10^{7} \Omega \mathrm{cm}$, it can be concluded that the peptide meets our expectation and functions as an insulator. Based on the confirmation that the peptide has a sufficiently large dielectric strength, the peptide insulator was 
deemed ready to be incorporated into an actual electronic device; for example, the TFT incorporated with the peptide insulator was tested.

\section{3. $\quad$ TFT characteristics}

The 3-D schematics, optical microscopic image, and transmission electron microscopy (TEM) cross-sectional image of the TFT fabricated as a possible application for the peptide insulator are shown in Fig. 2c-e, respectively.

The transfer curve is often used to characterize the performance of TFT by plotting the drain current as a function of gate voltage. From the transfer curves, device parameters were extracted and summarized in Table 1.

Due to absence of the peptide's quasi-static capacitance data, the mobility of the peptide-incorporated TFT was unobtainable, unfortunately. Still, comparison to the reference TFT with other parameters was able to be made. In terms of the subthreshold swing, the peptide TFT exhibited higher value compared to the reference TFT, and it was expected as the capacitance per unit area $\left(C_{\mathrm{i}}\right)$ of the peptide insulator is larger than that of the reference $\mathrm{SiO}_{2}$ owing to its huge dielectric constant. It is also notable that the threshold voltage $\left(V_{\text {th }}\right)$ of the peptide TFT shifts to more positive way as the $\mathrm{RH}$ decreases. This is probably due to charge trapping that occurs at the interface between the IGZO and the peptide insulator, and requires further study to find its origin.

Additionally, by comparing the performance between the peptide TFT and the reference $\mathrm{SiO}_{2}$ TFT in terms of the oncurrent and off-current levels, the on-current enhancement due to increased relative permittivity of the peptide insulator was confirmed. As shown in Fig. 6a, the large relative permittivity of the peptide insulator significantly enhanced the oncurrent of the peptide TFT. In Fig. 6b, the on-current values from the TFTs are shown and normalized with respect to the on current of the $\mathrm{SiO}_{2}$ TFT in $0 \% \mathrm{RH}$. The on-current of the peptide TFT was greater than that of the $\mathrm{SiO}_{2}$ TFT by 1.9 times, 35 times, and 102 times at the $\mathrm{RH}$ of $0 \%, 50 \%$, and $80 \%$, respectively. Additionally, it is important to note that the $\mathrm{SiO}_{2}$ used in the reference sample was thermally grown with the thickness of $100 \mathrm{~nm}$, which is $1 / 4$ of the thickness of the peptide insulator. It is believed that the peptide insulator can be utilized in many applications, such as displays or sensors to enhance the performance of the transistors by increasing their capacitance
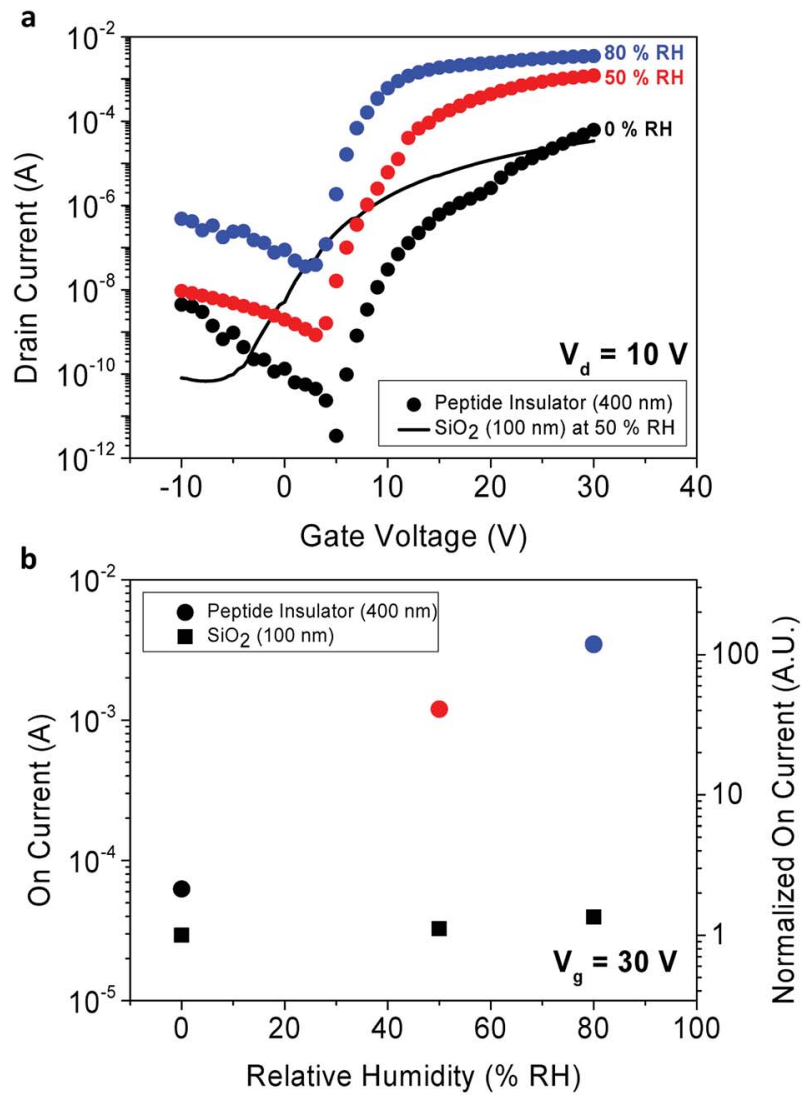

Fig. 6 Electrical characteristics of TFT samples. (a) Comparison of the transfer curves and (b) on-current level of the peptide TFT samples with the reference $\mathrm{SiO}_{2}$ samples in various humidity conditions.

significantly. In addition, the off-current also increased as the RH increased.

This tendency was illustrated in our earlier discussion about Fig. $5 \mathrm{~b}$, which showed the decreased resistivity of the peptide insulator in higher RH conditions. As shown in Fig. 6b, due to the increase in the off-current level in higher RH conditions, the on-off ratio decreased from $2.9 \times 10^{7}$ at $0 \% \mathrm{RH}$ to $1.4 \times 10^{6}$ at $50 \% \mathrm{RH}$, and to $1.2 \times 10^{5}$ at $80 \% \mathrm{RH}$. Even though both the on and off-current were increased, the on-off ratio of the peptide TFT exhibited a higher or similar value compared to that of the reference sample, which was $5.1 \times 10^{5}$ at $50 \% \mathrm{RH}$; this is due to the considerably increased on-current level of the peptide TFT.

Table 1 Electrical characteristics of TFT samples with different insulators ${ }^{a}$

\begin{tabular}{|c|c|c|c|c|}
\hline Insulator & $\mathrm{SiO}_{2}$ & Peptide & & \\
\hline Humidity & $50 \%$ RH & o\% RH & $50 \%$ RH & $80 \%$ RH \\
\hline$I_{\mathrm{on}}(\mathrm{A})$ & $3.4 \times 10^{-5}$ & $6.3 \times 10^{-5}$ & $1.2 \times 10^{-3}$ & $3.5 \times 10^{-3}$ \\
\hline$I_{\mathrm{on}} / I_{\mathrm{off}}$ & $5.0 \times 10^{-5}$ & $2.9 \times 10^{-7}$ & $1.4 \times 10^{-6}$ & $1.2 \times 10^{-5}$ \\
\hline Mobility $\left(\mathrm{cm}^{2} \mathrm{~V}^{-1} \mathrm{~s}^{-1}\right)^{*}$ & 3.01 & & & \\
\hline$V_{\text {th }}(\mathrm{V})$ & 4.75 & 21.76 & 9.82 & 6.18 \\
\hline
\end{tabular}

$a *$ Calculation of the mobility of the peptide-incorporated TFT was unable due to absence of the peptide's quasi-static capacitance. 
Additionally, in Table 2, the performance of the insulator and the peptide-incorporated TFT can be compared with the TFTs that used different conventional inorganic and organic insulator and channel materials. The peptide insulator showed the highest dielectric constant among different types of insulators and the TFT performance in terms of the on-off ratio was superior than the organic insulators while comparable to the inorganic insulators.

Since the transfer curves of the peptide TFT samples showed switching characteristics with the on-off ratio of over $10^{5}$ in all of the RH conditions, it can be concluded that the peptide insulator is successfully incorporated into the conventional IGZO TFT, and can be further utilized as a superior bio-induced dielectric material in conjunction with organic or even conventional inorganic electronic materials.

\section{Conclusion}

In conclusion, the peptide insulator was studied extensively to find the source of high relative permittivity. It was proven that the electrolytic behavior of the peptide insulator due to proton conduction is the major source of high relative permittivity. Although the peptide insulator suffers from relatively high leakage current and low dielectric constant at high frequency, a promising result was achieved by incorporating the peptide insulator into an oxide TFT as an alternate gate insulator, where the on-current level of the TFT was enhanced drastically due to the high relative permittivity of the peptide insulator, with the verification on the compatibility of the peptide insulator with the conventional inorganic materials. The peptide insulator is expected to be further utilized as a gate dielectric for lowfrequency and low-voltage operating devices, such as sensors, especially in conjunction with the aforementioned film-forming technology $y^{22}$ and further tuning of its chemical characteristics, as well as the electrical characteristics.

\section{Conflicts of interest}

The authors have no conflicts of interest to declare.

\section{Acknowledgements}

This work was supported by the Samsung Research Funding Center of Samsung Electronics under Project Number SRFCMA1401-51.

\section{Notes and references}

1 J. W. Chang, C. G. Wang, C. Y. Huang, T. D. Tsai, T. F. Guo and T. C. Wen, Adv. Mater., 2011, 23, 4077-4081.

2 M. Ambrico, P. F. Ambrico, A. Cardone, T. Ligonzo, S. R. Cicco, R. D. Mundo, V. Augelli and G. M. Farinola, Adv. Mater., 2011, 23, 3332-3336.

3 M. Irimia-Vladu, N. S. Sariciftci and S. Bauer, J. Mater. Chem., 2011, 21, 1350-1361. 
4 R. Capelli, J. J. Amsden, G. Generali, S. Toffanin, V. Benfenati, M. Muccini, D. L. Kaplan, F. G. Omenetto and R. Zamboni, Org. Electron., 2011, 12, 1146-1151.

5 J. Lee, I. R. Choe, Y. O. Kim, S. D. Namgung, K. Jin, H. Y. Ahn, T. Sung, J. Y. Kwon, Y. S. Lee and K. T. Nam, Advanced Functional Materials, 2017.

6 M. Amit, S. Appel, R. Cohen, G. Cheng, I. W. Hamley and N. Ashkenasy, Adv. Funct. Mater., 2014, 24, 5873-5880.

7 T. J. Peckham, J. Schmeisser and S. Holdcroft, J. Phys. Chem. $B, 2008,112,2848-2858$.

8 D. D. Ordinario, L. Phan, W. G. Walkup IV, J.-M. Jocson, E. Karshalev, N. Hüsken and A. A. Gorodetsky, Nat. Chem., 2014, 6, 596.

9 J. Wünsche, Y. Deng, P. Kumar, E. Di Mauro, E. Josberger, J. Sayago, A. Pezzella, F. Soavi, F. Cicoira and M. Rolandi, Chem. Mater., 2015, 27, 436-442.

10 P. Gascoyne, R. Pethig and A. Szent-Györgyi, Proc. Natl. Acad. Sci., 1981, 78, 261-265.

11 C. Zhong, Y. Deng, A. F. Roudsari, A. Kapetanovic, M. Anantram and M. Rolandi, Nat. Commun., 2011, 2, ncomms1489.

12 G. Wu, P. Feng, X. Wan, L. Zhu, Y. Shi and Q. Wan, Artificial Synaptic Devices Based on Natural Chicken Albumen Coupled Electric-Double-Layer Transistors, 2016.

13 S. Kelkar, K. Pandey, S. Agarkar, N. Saikhedkar, M. Tathavadekar, I. Agrawal, R. V. N. Gundloori and S. Ogale, ACS Sustainable Chem. Eng., 2014, 2, 2707-2714.

14 J. Stubbe, Curr. Opin. Chem. Biol., 2003, 7, 183-188.

15 V. K. Yachandra, K. Sauer and M. P. Klein, Chem. Rev., 1996, 96, 2927-2950.

16 P. A. Riley, Int. J. Biochem. Cell Biol., 1997, 29, 1235-1239.

17 H. Morgan, R. Pethig and G. Stevens, J. Phys. E: Sci. Instrum., 1986, 19, 80.

18 J. F. Nagle and S. Tristram-Nagle, J. Membr. Biol., 1983, 74, 114.
19 N. Agmon, Chem. Phys. Lett., 1995, 244, 456-462.

20 O. Larsson, E. Said, M. Berggren and X. Crispin, Adv. Funct. Mater., 2009, 19, 3334-3341.

21 R. B. Merrifield, J. Am. Chem. Soc., 1963, 85, 2149-2154.

22 H.-S. Jang, J.-H. Lee, Y.-S. Park, Y.-O. Kim, J. Park, T.-Y. Yang, K. Jin, J. Lee, S. Park and J. M. You, Nat. Commun., 2014, 5, 3665.

23 S. D. Namgung, J. Lee, I. R. Choe, T. Sung, Y.-O. Kim, Y.-S. Lee, K. T. Nam and J.-Y. Kwon, APL Mater., 2017, 5, 086109.

$24 \mathrm{~V}$. F. Lvovich, Impedance spectroscopy: applications to electrochemical and dielectric phenomena, John Wiley \& Sons, 2012.

25 R. A. Huggins, Ionics, 2002, 8, 300-313.

26 S. Herman, Delmar's standard textbook of electricity, Nelson Education, 2014.

27 D. K. Paul, R. McCreery and K. Karan, J. Electrochem. Soc., 2014, 161, F1395-F1402.

28 P. Zoltowski, J. Electroanal. Chem., 1998, 443, 149-154.

29 A. K. Jonscher, Universal relaxation law: a sequel to Dielectric relaxation in solids, Chelsea Dielectrics Press, 1996.

30 T. Kumazawa, M. Oishi and M. Todoki, IEEE Trans. Dielectr. Electr. Insul., 1994, 1, 133-138.

31 A. K. Jonscher, J. Phys. D: Appl. Phys., 1999, 32, R57.

32 K. H. Ji, J.-I. Kim, Y.-G. Mo, J. H. Jeong, S. Yang, C.-S. Hwang, S.-H. K. Park, M.-K. Ryu, S.-Y. Lee and J. K. Jeong, IEEE Electron Device Lett., 2010, 31, 1404-1406.

33 L. Lan and J. Peng, High-Performance Indium-Gallium-Zinc Oxide Thin-Film Transistors Based on Anodic Aluminum Oxide, 2011.

34 R. Parashkov, E. Becker, G. Ginev, T. Riedl, H.-H. Johannes and W. Kowalsky, J. Appl. Phys., 2004, 95, 1594-1596.

35 C. H. Wang, C. Y. Hsieh and J. C. Hwang, Adv. Mater., 2011, 23, 1630-1634. 